\title{
Mid-infrared Variability and Accretion in NGC 2264 Protostars
}

\author{
S. Terebey ${ }^{1}$, A. M. Cody ${ }^{2}$, L. M. Rebull ${ }^{3}$, and J. R. Stauffer ${ }^{3}$ \\ ${ }^{1}$ California State University Los Angeles; email: sterebe@calstatela.edu \\ ${ }^{2}$ NASA Ames Research Center; ${ }^{3}$ Spitzer Science Center; California Institute of Technology
}

\begin{abstract}
Variable mass accretion is thought to be an important aspect of protostar formation. Mid-infrared wavelength observations trace variations in accretion luminosity and thus can probe mass accretion on sub-AU scales. We present results from the Spitzer YSOVAR campaign towards Class I protostars in NGC 2264. The precise (0.02 mag) medium-cadence light curves at 3.6 and 4.5 microns show that young star variability is ubiquitous, with a variety of morphologies and time scales. A structure function analysis shows the light curves, on average, have a power-law behavior up to 30 days. The trend continues to longer timescales (years) for protostars (Class I), in contrast with the smaller brightness changes displayed by T Tauri stars (Class II). The power-law behavior suggests a stochastic process, such as turbulent mass accretion, drives the variability.
\end{abstract}

Keywords. stars:formation - stars:protostars - stars:variables: T Tauri

\section{Introduction and Data}

Young stars have long been recognized to be variable sources. The variability fits into a picture of a dynamic environment, where the inner disk feeds mass onto the star, and shocks dissipate energy thus leading to a variety of UV and optical phenomena. Protostars should share the same phenomena, although with lower stellar masses and higher accretion rates. However, protostars are enshrouded, so that optical and UV tracers are not accessible to study the inner disk, and thus motivate the need for mid-infrared studies. A key issue in star formation is understanding mass accretion. Variable mass accretion is important in providing a possible solution to the luminosity problem (e.g. Dunham et al. 2010).

In Orion, Morales-Calderon et al. (2011) found that $70 \%$ of stars with disks show mid-infrared variability. Extending to other clusters, and considering a data span of seven years, Rebull et al. (2014) find the mid-IR variability fraction scales with Class I content, which is used as a proxy for cluster age. For YSOs in NGC 2264, Cody et al. (2014) quantify a variety of light curve types, and demonstrate the kinds of variability that are fundamentally related to the presence of disks.

The current work focuses on characterizing the timescales of mid-infrared variability for protostars in NGC 2264. The analysis includes all available Spitzer $4.5 \mu \mathrm{m}$ data, from the cryogenic era (Sung et al. 2009), from $\sim 40$ days in 2010 (Rebull et al. 2014), and from $\sim 30$ days in 2011 (Cody et al. 2014). This young (2-4 Myr) cluster is located at a distance of $760 \mathrm{pc}$, contains about 1000 known members, and a similar number of candidate members (Park et al. 2000, Sung et al. 2009). Our protostar sample includes 37 Class I plus flat SED sources. The criteria for inclusion were conservative, and required 70 micron flux detection (e.g. Forbrich et al. 2010). For a comparison sample, we used 82 Class II sources from Cody et al. (2014). 

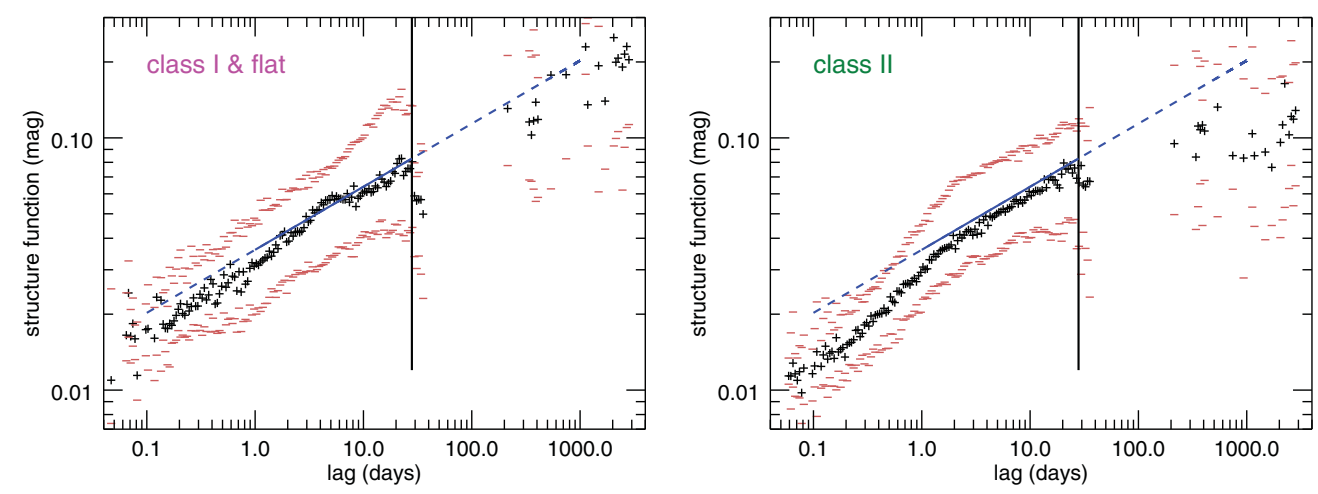

Figure 1. The $4.5 \mu \mathrm{m}$ ensemble Structure Function (crosses) shows the same power-law behavior (blue line) between 1 and 30 days for the Class I plus flat sample (left), and the Class II sample (right). At longer times ( 1000 days) the Class I plus flat sample approximately follows the same power-law trend (dashed blue line), whereas the Class II sample has 'turned over' at $0.1 \mathrm{mag}$, with the meaning that $0.1 \mathrm{mag}$ is the typical variability of the Class II sources. Red dashes show the inner 50 percent quartile range for objects contributing to the ensemble SF.

\section{Analysis and Conclusions}

We use the structure function $\operatorname{SF}(\Delta t)$ to characterize the light curves in a statistical sense. Similar to computing rms, it is an average of $\Delta m a g$ values, but is restricted to data points that have the same time lag $\Delta t=t_{i}-t_{j}$. For example, a non-variable object exhibits a SF that is roughly constant versus time lag. The ensemble SF is made from the median of the SF curves for each object. Examples of the SF in the context of quasar variability are given in Kelly et al. (2009) and Kozlowski et al. (2010).

The ensemble SF (Fig. 1) shows that two magnitude measurements separated by 10 days will, on average, differ by about 0.06 magnitudes for both samples, Class I plus flat (left panel) and and Class II (right panel). Moreover, both samples exhibit the same power-law slope of 0.25 between 1 and 30 days. However, at 1000 days the samples diverge: a typical Class I will change by $0.2 \mathrm{mag}$, but a Class II by only 0.1 mag.

Time scales of $\sim 10$ days at $4.5 \mu \mathrm{m}$ trace emission variability that occurs in the inner disk (0.1 AU). The power-law behavior exhibited between 1 and 30 days (Fig. 1) suggests a stochastic process such as turbulent mass-accretion is acting. The similar structure function found in the Class I and Class II samples implies similar physical processes are at work in the inner disk. At 1000 days, the increased variability of Class I objects suggests secular changes in the mass accretion rate. Future directions include the possibility of using mid-IR variability to determine disk mass accretion rates for protostars.

\section{References}

Cody, A. M., Stauffer, J. R., Baglin, A. et al. 2014, AJ, 147, 82

Dunham, M. M., Evans, Neal J., II, Terebey, S., et al. 2010, ApJ, 710, 470

Forbrich, J., Tappe, A., Robitaille, T., et al. 2010, ApJ, 716, 1453

Kelly, B. C., Bechtold, J., \& Siemiginowska, A. 2009, ApJ, 698, 895

Kozlowski, S., Kochanek, C. S., Stern, D., et al. 2010, ApJ, 716, 530

Morales-Calderon, M. , Stauffer, J. R., Hillenbrand, L. A. et al. 2011, ApJ, 733, 50

Park, B.-G., Sung, H, Bessell, M. S., \& Kang, Y. H. 2000, AJ, 120, 894

Rebull, L. R., Cody, A. M., Covey, K. R., et al. 2014, AJ, 148, 92

Sung, H, Stauffer, J. R. \& Bessell, M. S., 2009, AJ, 138, 1116 